\title{
Actor diversity (News Performance)
}

\section{AUTHOR}

Edda Humprecht

\section{KEYWORDS}

news coverage, journalism, diversity, integration, deliberation

FIELD OF APPLICATION/THEORETICAL FOUNDATION Analyses of actor diversity are theoretically linked to news performance and the democratic media function of integration (Imhof, 2010). This construct is related to the normative assumption that news content should represent society as a whole and thus cover a large variety of societal groups (Boydstun et al., 2014). More recent studies also focus on the influence of algorithms on news diversity (Möller et al., 2018).

Analyses are often carried out in three steps. First, all actors are (inductively or deductively) identified. Second, actors are coded according to predefined lists. Third, the level of diversity is determined using diversity indices (van Cuilenburg, 2007). Diversity indices are calculated at article level (internal diversity) or at the organizational level (external diversity) to compare diversity between news articles of a single outlet or between different news outlets.

\section{REFERENCES/COMBINATION WITH OTHER METHODS OF DATA COLLECTION}

Studies on actor diversity use both manual and automated content analysis to investigate the occurrence of actors and in texts. They use inductive or deductive approaches and/or a combination of both to identify actor categories and extend predefined lists of actors (van Hoof et al., 2014).

\section{EXAMPLE STUDIES:}

Masini et al. (2018); Humprecht \& Esser (2018)

\section{REFERENCES}

Boydstun, A. E., Bevan, S., \& Thomas, H. F. (2014). The importance of attention diversity and how to measure it. Policy Studies Journal, 42(2), 173-196. https://doi.org/10.1111/psj.12055

Humprecht, E., \& Esser, F. (2018). Diversity in Online News: On the importance of ownership types and media system types. Journalism Studies, 19(12), 1825-1847. https:// doi.org/10.1080/1461670X.2017.1308229

Imhof, K. (2010). Die Qualität der Medien in der Demokratie. In fög - Forschungsbereich Öffentlichkeit und Gesellschaft (Ed.), Jahrbuch 2010: Qualität der Medien Qualität der Medien (pp. 11-20). Schwabe. https:// doi.org/10.1007/978-3-322-97101-2_1

Masini, A., Van Aelst, P., Zerback, T., Reinemann, C., Mancini, P., Mazzoni, M., Damiani, M., \& Coen, S. (2018). Measuring and Explaining the Diversity of Voices and Viewpoints in the News: A comparative study on the determinants of content diversity of immigration news. Journalism Studies, 19(15), 2324-2343. https://doi. org/10.1080/1461670X.2017.1343650

Möller, J., Trilling, D., Helberger, N., \& van Es, B. (2018). Do not blame it on the algorithm: an empirical assessment of multiple recommender systems and their impact on content diversity. Information Communication and Society, 21(7), 959-977. https:// doi.org/10.1080/1369118X.2018.1444076

van Cuilenburg, J. (2007). Media Diversity, Competition and Concentration: Concepts and Theories. In E. de Bens (Ed.), Media Between Culture and Commerce (pp. 25-54). Intellect. 
van Hoof, A., Jacobi, C., Ruigrok, N., \& van Atteveldt, W. (2014). Diverse politics, diverse news coverage? A longitudinal study of diversity in Dutch political news during two decades of election campaigns. European Journal of Communication, 29(6), 668-686. https://doi.org/10.1177/0267323114545712

Table 1. Summary of studies on actor diversity.

\begin{tabular}{|c|c|c|c|c|}
\hline Author(s) & Sample & Unit of Analysis & Values & Reliability \\
\hline $\begin{array}{l}\text { Masini et al. } \\
(2018)\end{array}$ & $\begin{array}{l}\text { Content type: } \\
\text { news about } \\
\text { immigration } \\
\text { Outlet/ coun- } \\
\text { try: } 2 \text { news } \\
\text { outlets in four } \\
\text { countries (BE, } \\
\text { DE, IT, UK) } \\
\text { Sampling pe- } \\
\text { riod: January } \\
2013 \text { to April } \\
2014 \\
\text { Sample size: } \\
\text { N= 2490) }\end{array}$ & $\begin{array}{l}\text { Unit of analysis: news } \\
\text { article } \\
\text { No. of actors coded: max. } \\
10 \text { quoted or paraphrased } \\
\text { actors per article } \\
\text { Level of analysis: article } \\
\text { and news outlet level } \\
\text { Diversity measure: Sim- } \\
\text { pson's diversity index }\end{array}$ & $\begin{array}{l}\text { National politics, } \\
\text { international politics, } \\
\text { public opinion and } \\
\text { ordinary people, im- } \\
\text { migrants, civil socie- } \\
\text { ty, public agencies/ } \\
\text { organizations, judi- } \\
\text { ciary/police/military, } \\
\text { religion, business/ } \\
\text { corporate/finance, } \\
\text { journalists/ media ce- } \\
\text { lebrities, traffickers/ } \\
\text { smugglers }\end{array}$ & $\begin{array}{l}\text { Krippen- } \\
\text { dorff's alpha } \\
\text { average } \\
\geq 0.78\end{array}$ \\
\hline $\begin{array}{l}\text { Humprecht } \\
\text { \& Esser } \\
(2018)\end{array}$ & $\begin{array}{l}\text { Content type: } \\
\text { Political rou- } \\
\text { tine-period } \\
\text { news } \\
\text { Outlet/ } \\
\text { country: } 48 \\
\text { online news } \\
\text { outlets from } \\
\text { six countries } \\
\text { (CH, DE, FR, } \\
\text { IT, UK, US) } \\
\text { Sampling } \\
\text { period: June - } \\
\text { July } 2012 \\
\text { Sample size: } \\
\text { N= } 1660\end{array}$ & $\begin{array}{l}\text { Unit of analysis: Political } \\
\text { news items (make refe- } \\
\text { rence to a political actor, } \\
\text { e.g. politician, party, } \\
\text { institution in headline, } \\
\text { sub-headline, in first } \\
\text { paragraph or in an ac- } \\
\text { companying visual) } \\
\text { News items are all jour- } \\
\text { nalistic articles mentio- } \\
\text { ned on the front page } \\
\text { ('first layer' of the web- } \\
\text { site) that are linked to the } \\
\text { actual story (on second } \\
\text { layer of website) } \\
\text { No. of actors coded: Max. } \\
5 \text { main actors (mentio- } \\
\text { ned twice) per news item } \\
\text { measured } \\
\text { Level of analysis: news } \\
\text { outlet level } \\
\text { Diversity measure: relative } \\
\text { entropy }\end{array}$ & $\begin{array}{l}\text { Executive (head of } \\
\text { state and national go- } \\
\text { vernment), legislative } \\
\text { (national parliament } \\
\text { and national parties), } \\
\text { judicial (national } \\
\text { courts and judges), } \\
\text { national administ- } \\
\text { ration (prosecution, } \\
\text { regional government } \\
\text { authority, and poli- } \\
\text { ce or army), foreign } \\
\text { politicians (foreign } \\
\text { heads of state and } \\
\text { other foreign politi- } \\
\text { cians), and interna- } \\
\text { tional organizations } \\
\text { (supranational and } \\
\text { international organi- } \\
\text { zations) }\end{array}$ & $\begin{array}{l}\text { Cohen's kap- } \\
\text { pa average } \\
\geq 0.76\end{array}$ \\
\hline
\end{tabular}

